\title{
A novel de novo frame-shift mutation of the $E D A$ gene in a Chinese Han family with hypohidrotic ectodermal dysplasia
}

\author{
Changzheng Huang • Qinbo Yang $\cdot$ Tie Ke $\cdot$ Haisheng Wang • \\ Xu Wang · Jiqun Shen $\cdot$ Xin Tu $\cdot$ Jin Tian · Jing Yu Liu • \\ Qing K. Wang $\cdot$ Mugen Liu
}

Received: 3 August 2006/ Accepted: 1 September 2006/ Published online: 26 October 2006

(C) The Japan Society of Human Genetics and Springer 2006

\begin{abstract}
Hypohidrotic ectodermal dysplasia (HED) is characterized by severe hypohidrosis, hypotrichosis, and hypodontia. It can be inherited in autosomal dominant, autosomal recessive, or X-linked patterns. Mutations in the EDA gene, which encodes ectodysplasin-A, are responsible for X-linked HED (XLHED).
\end{abstract}

Changzheng Huang and Qinbo Yang contribute equally to this work.

\footnotetext{
C. Huang $\cdot$ Q. Yang $\cdot$ T. Ke $\cdot$ X. Wang $\cdot$ J. Shen .

X. Tu · J. Y. Liu . Q. K. Wang ( $\square) \cdot$ M. Liu ( $\square)$

Center for Human Genome Research,

Huazhong University of Science and Technology,

Wuhan, Hubei 430074, China

e-mail: lium@mail.hust.edu.cn

Q. K. Wang

e-mail: qkwang@mail.hust.edu.cn

C. Huang $\cdot$ J. Tian

Department of Dermatology,

Union Hospital, Tongji Medical College,

Huazhong University of Science and Technology,

Wuhan, Hubei 430022, China

Q. Yang $\cdot$ T. Ke $\cdot$ X. Wang $\cdot$ J. Shen $\cdot$ X. Tu .

J. Y. Liu · Q. K. Wang · M. Liu

College of Life Science and Technology,

Huazhong University of Science and Technology,

Wuhan, Hubei 430074, China

H. Wang

Institute of Forensic Science and Technology,

Bureau of Hubei Public Security,

Wuhan, Hubei 430070, China

Q. K. Wang

Department of Molecular Cardiology,

Lerner Research Institute, Cleveland Clinic,

Cleveland, OH 44195, USA
}

In the present study, we identified a Chinese Han family with XLHED. Direct DNA sequence analysis of the entire coding region and exon-intron boundaries of $E D A$ identified a novel de novo mutation, c.573_574insT, in two affected males and one carrier female. Restriction fragment length polymorphism (RFLP) analysis showed that the mutation was not present in 200 controls. The 1-bp insertion mutation resulted in a frameshift, which causes premature termination of EDA polypeptide and truncation of the EDA protein. These results suggest that the c.573_574insT mutation of the EDA gene is a cause for XLHED in the family. To the best of our knowledge, this is the first de novo insertion mutation of $E D A$ described for XLHED.

Keywords EDA - Frameshift mutation - RFLP . $\mathrm{X}$-linked hypohidrotic ectodermal dysplasia

\section{Introduction}

Hypohidrotic ectodermal dysplasia (HED) is found to occur worldwide, with an estimated incidence of 1 per 100,000 births (Zonana 1993). Ectodermal dysplasias typically affect the hair, teeth, nails, and/or skin. HED is primarily characterized by the partial or complete absence of certain sweat glands (eccrine glands), causing the lack of or diminished sweating (anhidrosis or hypohidrosis), heat intolerance, and fever, abnormally sparse hair (hypotrichosis), and the absence (anodontia or hypodontia) and/or malformation of certain teeth. It can be inherited in autosomal dominant, autosomal recessive, or X-linked patterns. However, X-linked HED (XLHED; OMIM 305100) 
is the most common form of HED (Pinheiro and Freire-Maia 1994). If unrecognized, XLHED is one of the causes of fever of unknown origin, repeated bronchitis, and sudden death during infancy and early childhood (Zhang et al. 2003).

XLHED is caused by mutations in the EDA gene (Kere et al. 1996), which encodes ectodysplasin-A, a type II transmembrane protein of 391 amino acids. Ectodysplasin-A has a furin site, a collagen-like sequence in the linker region between the transmembrane helix and the tumor necrosis factor (TNF) ligand motif (Schneider et al. 2001; Wisniewski et al. 2003). Ectodysplasin-A is involved in the regulation of ectodermal morphogenesis. By specific binding to the ectodysplasin receptor (EDAR), a member of the TNF receptor family, EDA may activate the NF- $\kappa \mathrm{B}$ and cJun N-terminal kinase pathways (Yan et al. 2000). Mutations in EDAR (Chassaing et al. 2006), EDAR$A D D$ (Cui et al. 2002), or NEMO (Vinolo et al. 2006) are also reported to be associated with anhidrotic ectodermal dysplasia.

In this study, we studied a Chinese Han family with XLHED. Using linkage analysis, we mapped the disease locus to chromosome Xq12-q13, where the EDA gene is located. By direct DNA sequence analysis, we identified a novel de novo 1-bp insertion (c.573574insT) in exon 4 of EDA that causes XLHED.

\section{Subjects and methods}

Study participants and the isolation of genomic DNA

We analyzed a Chinese Han family with XLHED (Fig. 1a). The proband (III-1) was a 21-year-old male (Fig. 1b). He had typical triad of the disorder, including hypohidrosis, hypotrichosis, and anodontia, and suffered from recurrent idiopathic fever, which was more frequent and more obvious in the summer and during eating. His scalp hair and eyelashes were sparse, thin, and dry, and his eyebrows were scanty to absent. Sparse hair distributions were also noted in the beard and pubic areas, axillae, and trunk. He also had characteristic facial appearance, including a prominent forehead, high and wide cheekbones and narrow lower half of the face, a depressed nasal bridge, large and conspicuous nostrils, thickened lips, and a large chin. Yellow-brown hyperpigmentation was observed around the periorbital area, nose, and mouth. He was found totally anodontia since his birth. However, his physical development was normal, and no abnormalities in his finger and toe nails were identified. His younger brother

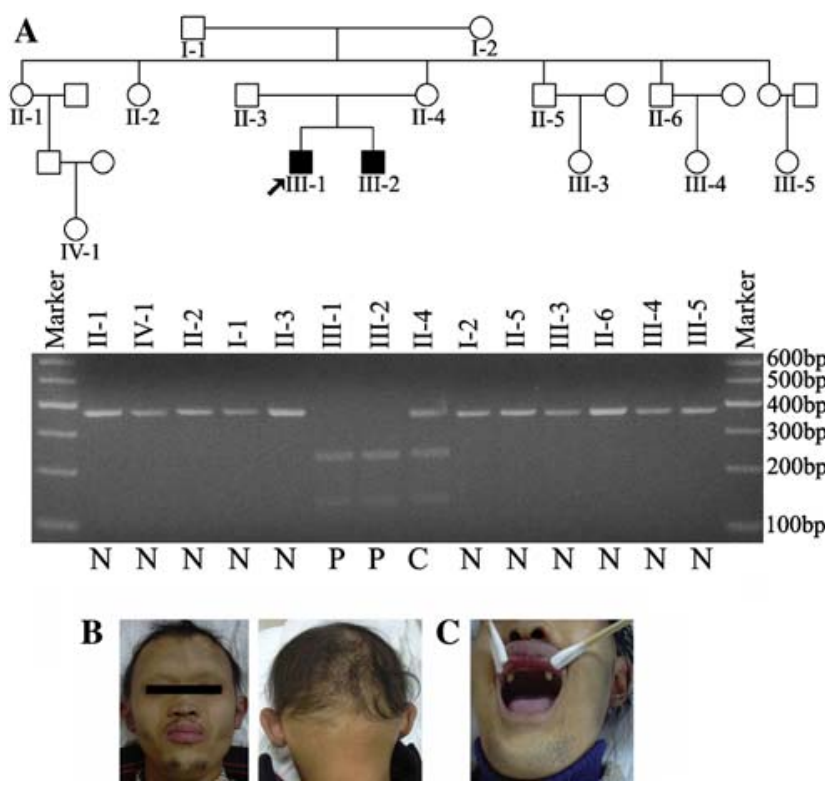

Fig. 1 a The pedigree structure and results of the restriction fragment length polymorphism (RFLP) analysis of a Chinese family with X-linked hypohidrotic ectodermal dysplasia (XLHED). RFLP analysis showed that the 1-bp insertion cosegregated with two affected individuals, III-1 and III-2, who were hemizygous for the c.573-574insT mutation. The female carrier (II-4) was heterozygous for the mutation, and other normal family members carried the wild type allele. Note that the wild type allele is represented by the 368-bp DNA band, and the mutant allele is shown as two bands of 237 and 132-bp in sizes, respectively. b Clinical features of the affected individual III-1 in the Chinese family (21-year-old male with hypohidrosis, hypotrichosis, and anodontia). The patient appeared to be prematurely aged. c Clinical features of the affected individual III-2 (19-year-old male with similar symptoms and clinical onsets as III-1, except that he has two conical-shaped teeth)

(III-2) had similar symptoms and clinical onsets, except that he had two conical-shaped teeth (Fig. 1c).

Informed consent was obtained from each participating family member or their legal guardian. Genomic DNA was extracted from the peripheral blood with Wizard Genomic DNA Purification Kit (Promega, Madison, WI).

\section{Linkage analysis}

The possible involvement of the EDA gene in the HED family was initially examined by linkage analysis with markers DXS991 and DXS986. Genotyping was carried out using an ABI 3100 Genetic Analyzer and allele-typing was performed using the GeneMapper 2.5 software (Applied Biosystems, Foster City, CA).

DNA sequence analysis and mutation detection

The entire coding region and exon-intron boundaries of EDA were PCR-amplified and sequenced as de- 
scribed previously (Wang et al. 2005). The primers used are shown in Table 1.

\section{RFLP analysis}

Since mutation c.573-574insT generated a novel Nco I restriction enzyme site (CCATGG) in exon 4 of the $E D A$ gene, we PCR-amplified exon 4 and the flanking sequences from all family members and 200 normal controls. The PCR products were digested with Nco I (TaKaRa, Dalian, China) at $37^{\circ} \mathrm{C}$ for $4 \mathrm{~h}$, and separated on a $2 \%$ agarose gel.

Paternal testing

To confirm the familial relationship of the mutation carrier with her parents, we performed genotyping analysis using a set of informative microsatellite markers from various chromosomes, including D8S1179, D21S11, D7S820, D3S1358, D13S317, D16S539, D2S1338, D19S43, D18S51, D5S818, CSF1PO, AMEL, $F G A, T P O X$, THO1, and $v W A$. Transmission analysis for each marker was then carried out.

\section{Results}

Linkage analysis of the Chinese XLHED family with two markers on chromosome $\mathrm{X}$ suggested that the $E D A$ gene was responsible for the disease phenotype (data not shown). Direct DNA sequence analysis identified a novel 1-bp insertion, c.573-574insT, in exon 4 of EDA (GenBank accession number NM_001399) (Fig. 2). This insertion resulted in a frame-shift that causes replacement of the C-terminal 200 amino acids of EDA (starting with amino acid residue $\mathrm{G}_{192}$ ) with a short polypeptide with 47 unrelated amino acid residues. The predicted mutant protein lacks the part of the collagen domain and the entire TNF-homology domain.

Direct DNA sequence and RFLP analyses showed that the 1-bp insertion co-segregated with HED in the family (Fig. 1a). Further RFLP analysis did not detect the c.573-574ins mutation in 200 normal controls. These results suggested that the c.573-574insT mutation of $E D A$ is not a rare polymorphism, but a causative mutation for XLHED in the Chinese Han family.

We did not find the c.573-574insT mutation from individuals I-1 and I-2, but their daughter, individual II-4 in the family, carried the 1-bp insertion, and transmitted this mutation to her two sons (Fig. 1a). Paternal testing with multiple informative microsatellite markers from various chromosomes confirmed the familial relationship of individual II-4 with both parents. These results demonstrate that the c.573-574insT insertion is a de novo mutation.

\section{Discussion}

In this study, we identified a novel 1-bp insertion, c.573-574insT, in the EDA gene in a Chinese Han family with XLHED. The mutation occurred in two affected males as a hemizygous state, and in the carrier female as a heterozygous state. It was not present in other normal family members or 200 normal controls. Furthermore, the mutation arose de novo. These results strongly suggest that the c.573-574insT mutation
Table 1 Primers used for polymerase chain reaction (PCR) amplification of the human ectodysplasin-A $(E D A)$ gene

\begin{tabular}{|c|c|c|}
\hline Exon & Sequence $\left(5^{\prime}-3^{\prime}\right)$ & $\begin{array}{l}\operatorname{Tm}\left({ }^{\circ} \mathrm{C}\right) \\
\text { fragment (bp) }\end{array}$ \\
\hline \multirow[t]{2}{*}{ Exon1-1 } & Forward: AGAGGTCGTGAACGGCTGAGG & \multirow[t]{2}{*}{$54 ; 264$} \\
\hline & Reverse: CGCAACTCTAGGTAGCAGCACAAC & \\
\hline \multirow[t]{2}{*}{ Exon1-2 } & Forward: GCCTGCTCTTCCTGGGTTTCTT & \multirow[t]{2}{*}{$54 ; 400$} \\
\hline & Reverse: TCССТGGTCСТGСССТСТАААТ & \\
\hline \multirow[t]{2}{*}{ Exon2 } & Forward: GCTGGTTTTTTATGTTGGCTATGAC & \multirow[t]{2}{*}{$52 ; 266$} \\
\hline & Reverse: CCACCATGCCCTACCAAGAAG & \\
\hline \multirow[t]{2}{*}{ Exon3 } & Forward: TTTGCAGTGTCTTGGGGATCC & \multirow[t]{2}{*}{$53 ; 346$} \\
\hline & Reverse: GCAGGGAGAAGAACAAGGAAGAAT & \\
\hline \multirow[t]{2}{*}{ Exon4 } & Forward: CCGAGATCGTGCCACTGAACT & \multirow[t]{2}{*}{$53 ; 368$} \\
\hline & Reverse: CCCCATCTCCACCGTTTGAA & \\
\hline \multirow[t]{2}{*}{ Exon5 } & Forward: TCAGGTGAGGGGAAAAGGAAGT & \multirow[t]{2}{*}{$52 ; 240$} \\
\hline & Reverse: GGGCTGTGAGTGAAAACCGTC & \\
\hline \multirow[t]{2}{*}{ Exon6 } & Forward: AGGGGAGAGGGATCAGAATTG & \multirow[t]{2}{*}{$50 ; 256$} \\
\hline & Reverse: AGGCTGGGTGATTATTTGGAG & \\
\hline \multirow[t]{2}{*}{ Exon7 } & Forward: TGCCTCGATTATTCTGACATGTACTG & \multirow[t]{2}{*}{$53 ; 300$} \\
\hline & Reverse: CCCAAAGCAGGAAGTTAGCCATT & \\
\hline \multirow[t]{2}{*}{ Exon8 } & Forward: CСССАСССТСТСТТТССТСТСТТС & \multirow[t]{2}{*}{$56 ; 412$} \\
\hline & Reverse: GGCTGCAACACCAATACACCTCAC & \\
\hline
\end{tabular}


Fig. 2a-d Identification of a novel 1-bp insertion in the $E D A$ gene. a DNA sequence of exon 4 of the $E D A$ gene from a normal male (II-3 in Fig. 1a). b DNA sequence from an affected male (III-1) showing the c.573-574insT mutation. c Normal cDNA and protein sequences of the EDA collagen domain. d The c.573-574insT mutation resulted in a frame-shift, leading to replacement of the C-terminal 200 amino acids of EDA with an aberrant polypeptide with 47 unrelated amino acids. The insertion occurs at codon 192

\section{a}

Normal male

C C C TCCAGGACCCCAGGACCTCCAGG

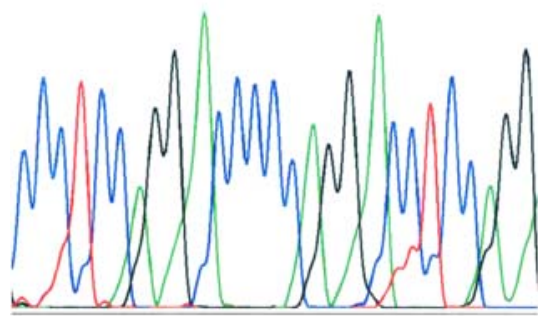

b
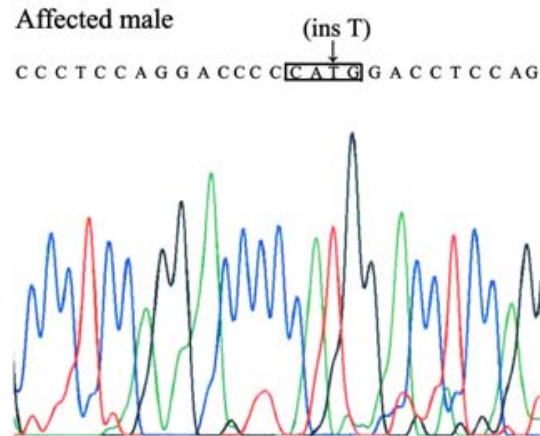

c

Normal EDA cDNA and amino acid sequence

GGA AAG AAA GCA GGA CCT CCT GGA CCC AAT GGC CCT

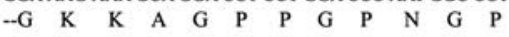
CCA GGA CCC CCA GGA CCT CCA GGA COC CAG GGA CCC $\begin{array}{llllllllllllll} & P & G & P & P & G & P & P & G & P & Q & G & P\end{array}$ CCA GGA ATT CCA GGG ATT CCT GGA ATT CCA GGA ACA $\begin{array}{llllllllllll}P & G & I & P & G & I & P & G & \text { I } & \text { P } & G & T\end{array}$ ACT GTT ATG TTA CCA CCT GGT CCT CCA GGT CCT CCT $\begin{array}{llllllllllll}T & V & M & G & P & P & G & P & P & G & P & P\end{array}$ GGT CCT CAA GGA CCC CCT GGA CTA CAG GGA CCT TCT

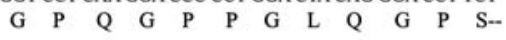
GGT GCT GCT GAT-

G A A D -

d

Mutant EDA cDNA and amino acid sequence

GGA AAG AAA GCA GGA CCT CCT GGA CCC AAT GGC CCT $\begin{array}{lllllllllll}-G & K & K & A_{\text {ing } T} \text { G } & P & P & G & P & N & G & P\end{array}$ CCA GGA CCC CCA TGG ACC TCC AGG ACC CCA GGG ACC $\begin{array}{llllllllllll}\text { P } & G & \text { P } & \text { P } & \text { W } & \text { T } & \text { S } & \text { R } & \text { T } & \text { P } & \text { G } & \text { T }\end{array}$ CCC AGG AAT TCC AGG GAT TCC TGG AAT TCC AGG AAC

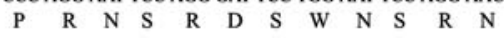
AAC TGT TAT GTT ACC ACC TGG TCC TCC AGG TCC TCC $\begin{array}{llllllllllll}N & C & Y & G & T & T & W & S & S & R & S & S\end{array}$ TGG TCC TCA AGG ACC CCC TGG ACT ACA GGG ACC TTC $\begin{array}{llllllllllll}\text { W } & \text { S } & \text { S } & \text { R } & \text { T } & \text { P } & \text { W } & \text { P } & \text { P } & \text { G } & \text { T } & \text { F }\end{array}$ TGG TGC TGC TGA

W $\quad \mathrm{C} \quad \mathrm{C} *$ causes the disease in the family, and it represents the first de novo insertion identified in the EDA gene.

The ectodysplasin-A protein contains several domains: a small N-terminal intracellular domain followed by the transmembrane domain, and a larger C-terminal extracellular domain, which contains a furin site, a collagen-like domain (containing 19 Gly-X-Y repeats), and a TNF homology domain. The furin domain functions as a cleavage site for a furin protease. The collagen domain is crucial for multimerization of EDA trimers. The TNF homology domain consists of 10 predicted anti-parallel $\beta$-sheets linked by variable loops, as in other members of the TNF family, and is necessary for the homotrimerization of ligands and its binding to the receptor (Ezer et al. 1999). The c.573574ins T insertion resulted in a frame-shift that truncates the EDA protein by 200 amino acid residues. The resultant mutant protein lacks the part of the collagenlike domain and the TNF-homology domain. Thus, the 1-bp insertion may significantly affect the function(s) of the EDA protein by disrupting two important functional domains.

Recently, one novel R65G mutation in the EDA gene was identified in a Mongolian family with only hypodontia, and without other HED manifestations (Tao et al. 2006), whereas another mutation G291R was reported in a Japanese family with hypohidrosis, hypotrichosis, and hypodontia (with only a primary central incisor and a permanent central incisor bud in the maxilla) (Sekiguchi et al. 2005), suggesting phenotypic heterogeneity of this syndrome. It is interesting to note that heterozygous mutation carriers of XLHED may have variable clinical features, displaying minor or moderate degrees of hypodontia, hypotrichosis, and hypohidrosis. In some families, the obligated carriers did not exhibit any clinical phenotype. The affected members of the family studied here displayed severe EDA symptoms of hypodontia (anodontia), hypotrichosis, and hypohidrosis, but these clinical features were not demonstrated in their heterozygous mother.

Acknowledgments We would like to thank the members of the Chinese family for their participation in this study. This work was supported by the Tenth "Five-Year" National Science and Technology Key Program of China grant no. 2004BA720A (to M. L.), the National Natural Science Foundation of China grant no. 30571677 (to M. L.), and the Chinese Ministry of Science and Technology National High Technology "863" Programs of China grant no. 2002BA711A07 (to Q. K. W.).

\section{References}

Chassaing N, Bourthoumieu S, Cossee M, Calvas P, Vincent MC (2006) Mutations in EDAR account for one-quarter of nonED1-related hypohidrotic ectodermal dysplasia. Hum Mutat 27(3):255-259

Cui CY, Durmowicz M, Tanaka TS, Hartung AJ, Tezuka T, Hashimoto K, Ko MS, Srivastava AK, Schlessinger D (2002) EDA targets revealed by skin gene expression profiles of 
wild-type, Tabby and Tabby EDA-A1 transgenic mice. Hum Mol Genet 11(15):1763-1773

Ezer S, Bayes M, Elomaa O, Schlessinger D, Kere J (1999) Ectodysplasin is a collagenous trimeric type II membrane protein with a tumor necrosis factor-like domain and colocalizes with cytoskeletal structures at lateral and apical surfaces of cells. Hum Mol Genet 8(11):2079-2086

Kere J, Srivastava AK, Montonen O, Zonana J, Thomas N, Ferguson B, Munoz F, Morgan D, Clarke A, Baybayan P, Chen EY, Ezer S, Saarialho-Kere U, de la Chapelle A, Schlessinger D (1996) X-linked anhidrotic hypohidrotic ectodermal dysplasia is caused by mutation in a novel transmembrane protein. Nat Genet 13(4):409-416

Pinheiro M, Freire-Maia N (1994) Ectodermal dysplasias: a clinical classification and a causal review. Am J Med Genet 53(2):153-162

Schneider P, Street SL, Gaide O, Hertig S, Tardivel A, Tschopp J, Runkel L, Alevizopoulos K, Ferguson BM, Zonana J (2001) Mutations leading to X-linked hypohidrotic ectodermal dysplasia affect three major functional domains in the tumor necrosis factor family member ectodysplasin-A. J Biol Chem 276(22):18819-18827

Sekiguchi H, Wang XJ, Minaguchi K, Yakushiji M (2005) A point mutation of the ED1 gene in a Japanese family with X-linked hypohidrotic ectodermal dysplasia. Int J Paediatr Dent 15(1):73-77

Tao R, Jin B, Guo SZ, Qing W, Feng GY, Brooks DG, Liu L, Xu J, Li T, Yan Y, He L (2006) A novel missense mutation of the EDA gene in a Mongolian family with congenital hypodontia. J Hum Genet 51(5):498-502
Vinolo E, Sebban H, Chaffotte A, Israel A, Courtois G, Veron M, Agou F (2006) A point mutation in NEMO associated with anhidrotic ectodermal dysplasia with immunodeficiency pathology results in destabilization of the oligomer and reduces lipopolysaccharide- and tumor necrosis factormediated NF-kappa B activation. J Biol Chem 281(10):6334-6348

Wang Q, Liu M, Xu C, Tang Z, Liao Y, Du R, Li W, Wu X, Wang X, Liu P, Zhang X, Zhu J, Ren X, Ke T, Wang Q, Yang J (2005) Novel CACNA1S mutation causes autosomal dominant hypokalemic periodic paralysis in a Chinese family. J Mol Med 83(3):203-208

Wisniewski SA, Kobielak A, Trzeciak WH, Kobielak K (2003) Recent advances in understanding of the molecular basis of anhidrotic ectodermal dysplasia: discovery of a ligand, ectodysplasin A and its two receptors. J Appl Genet 43(1):97-107

Yan M, Wang LC, Hymowitz SG, Schilbach S, Lee J, Goddard A, de Vos AM, Gao WQ, Dixit VM (2000) Two-amino acid molecular switch in an epithelial morphogen that regulates binding to two distinct receptors. Science 290(5491):523-527

Zhang XJ, Chen JJ, Song YX, Yang S, Xiong XY, Zhang AP, He PP, Gao M, Li YB, Lin D, Huang W (2003) Mutation analysis of the ED1 gene in two Chinese Han families with $\mathrm{X}$-linked hypohidrotic ectodermal dysplasia. Arch Dermatol Res 295(1):38-42

Zonana J (1993) Hypohidrotic (anhidrotic) ectodermal dysplasia: molecular genetic research and its clinical applications. Semin Dermatol 12(3):241-246 